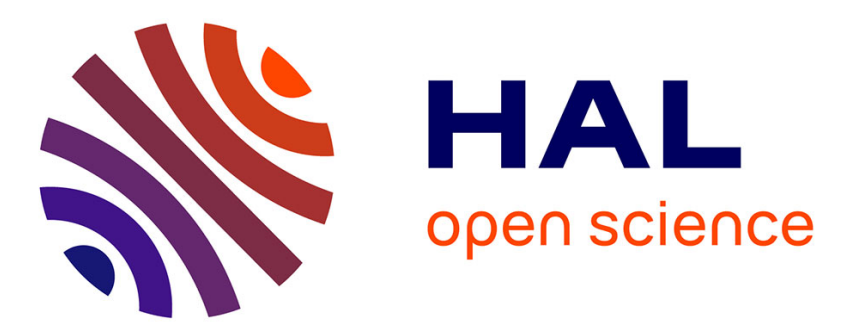

\title{
An extension of the target theory in biology applied to system reliability \\ Thierry Bastogne, Pierre Vallois
}

\section{To cite this version:}

Thierry Bastogne, Pierre Vallois. An extension of the target theory in biology applied to system reliability. Fouad El Ouardighi and Konstantin Kogan. Models and Methods in Economics and Management Science: Essays in Honor of Charles S. Tapiero, Springer, pp.155-181, 2014, International Series in Operations Research \& Management Science, 978-3-319-00668-0. hal-00911872

\section{HAL Id: hal-00911872 https://hal.science/hal-00911872}

Submitted on 30 Nov 2013

HAL is a multi-disciplinary open access archive for the deposit and dissemination of scientific research documents, whether they are published or not. The documents may come from teaching and research institutions in France or abroad, or from public or private research centers.
L'archive ouverte pluridisciplinaire HAL, est destinée au dépôt et à la diffusion de documents scientifiques de niveau recherche, publiés ou non, émanant des établissements d'enseignement et de recherche français ou étrangers, des laboratoires publics ou privés. 


\title{
An extension of the target theory in biology applied to system reliability
}

\author{
Thierry Bastogne ${ }^{(1)}$, Pierre VALLOIS $^{(2)}$
}

May 23, 2012

(1) Université de Lorraine, Centre de Recherche en Automatique de Nancy (CRAN), INRIA-BIGS, CNRS UMR 7039, BP 239, F-54506 Vandœuvre-lès-Nancy Cedex, France

(2) Université de Lorraine, Institut de Mathématiques Elie Cartan, INRIA-BIGS, CNRS UMR 7502,

BP 239, F-54506 Vandœuvre-lès-Nancy Cedex, France

\begin{abstract}
We consider rough products produced by a factory. Each product coming from the plant has $m$ vital elements and some elements can be damaged. To obtain a perfect product (i.e. all the constitutive $m$ elements are safe) all the damaged elements are repaired and a test phase follows. The result of this two-steps procedure is random. We suppose that the number $Z_{k}$ of non-damaged elements is a Markov chain valued in the set $\{0,1, \cdots, m\}$, where $k$ is the number of applied repairingtest phases. We have a qualitative result which says that if the repair phase is efficient then $P\left(Z_{k}=m\right)$ is close to 1 . As for production of a large number $n$ of products, the former result allows us to give conditions under which either the $n$ elements or a fraction of these $n$ elements are (is) safe after the application of $k$ previous maintenance phases.
\end{abstract}

Key words and phrases : Reliability, repairing procedure, target theory, treatment of cancer by radiotherapy, damaged cell, Markov chain, large deviations.

AMS 2000 subject classifications : 60 F 10, 60 F 05, 60 J 10, 60 J 20, 60 K 10.

\section{Introduction}

Most engineering systems endure degradations with time from wear, manufacturing defects, shocks and damages, and ultimately fail when the total damage exceeds a failure critical level [11]. Such events are usually mathematically described as stochastic processes and different models have been developed to analyze reliability properties and improve maintenance policies. In this chapter, we propose a bio-inspired modeling of engineering systems reliability, based on the target theory and hit-modeling paradigm introduced in the 1920s when biologists were beginning to develop quantum approaches to inactivation phenomena in irradiated biological tissue $[8,4,5,1,10,14,13]$.

1.1 In target theory, a cell is assumed to get different vital sites called targets which must be all inactivated to kill the cell. For instance, it is accepted that the chromosomes are sensitive targets but there is additional evidence that the nuclear membrane, or some cell organelles close to the nuclear membrane can also be regarded as targets. Each target is deactivated when it is hit by a number of radiation particles. There are several classes of hit models classified by the number of targets and the number of hits. In these models, it is generally assumed that cells have an homogeneous behavior. In practice, there are at least three main reasons to put this hypothesis into question. The first cause of heterogeneity comes from the nonuniform spatial distribution of the radiation dose. The second cause is due to the differences between the cell types (necrotic, quiescent, proliferating, stem cells, etc.) and 
the nonuniform concentration of oxygen and nutrients. A third factor corresponds to a cell-to-cell variability of damages and to the variation of the cell sensitivity to radiation. In [2], a model of tumor growth based on the target and hit modeling paradigm has been proposed. It is based on a Markov chain formalism able to describe both the target reparation between two consecutive dose fractions of the radiation schedule and the heterogeneity of damages induced by radiations.

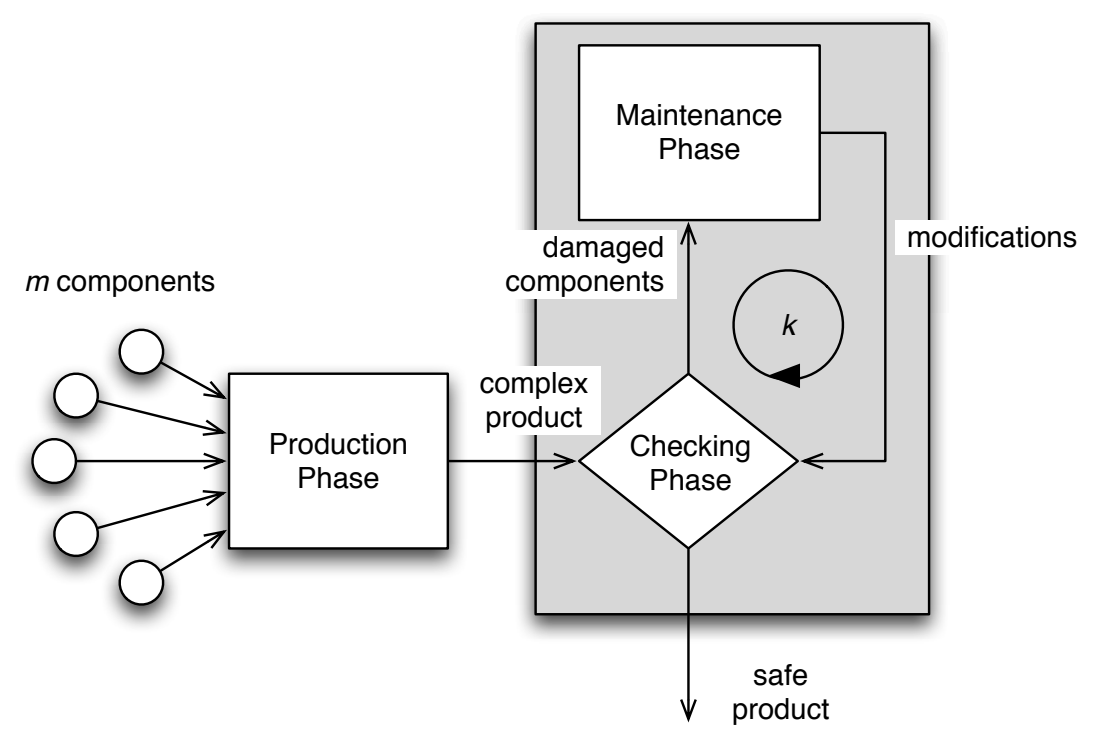

Figure 1: Reliability test schedule of a manufacturing system based on a loop composed of a checking phase and a maintenance step. $k$ is the number of maintenance cycles in the loop.

1.2 We claim that this model may be applied to other fields than cell biology, for instance reliability of engineering systems. For instance, let us consider a space launcher composed of numerous subsystems such as the payload structure, the guidance part, and the propulsion system, which are themselves made up of thousands of constitutive elements. The production of such sophisticated systems is not perfect and the products can have different manufacturing faults. If the $m$ components of a given product have no failure, the product can be directly used or sold. Otherwise, if at least one of its $m$ constitutive elements is out of order, the product has to be repaired. As illustrated in Figure 1, the maintenance procedure is iteratively repeated until each product is completely safe. We assume that the time period of the maintenance cycle is constant and the number of cycles for each product is noted $k$, which is equivalent to a discrete time. $k=0$ corresponds to the time instant when the product is coming out from manufacturing and has undergo no maintenance. During the maintenance cycle, two successive random phenomena can occur.

1. Reparation phase. At the beginning of the $k$-th cycle, all the $m$ elements of a product are checked. If $i \geq 1$ elements are detected as damaged then each element is repaired. The repair mechanism only concerns these $i$ elements. However the result of a repair of a given element is not certain, we suppose that it is successful with probability $r$ and the damaged element remains in the same state with probability $1-r$. The results concerning the maintenance of these $i$ elements are supposed to be independent. If we adopt the convention that a disk with (without) a cross denotes a damaged (resp. safe) element, the previous procedure can be summarized as

$$
\otimes \stackrel{r}{\longrightarrow} \bigcirc \quad \otimes \stackrel{1-r}{\longrightarrow} \otimes \quad \bigcirc \stackrel{1}{\longrightarrow} \bigcirc .
$$


2. Damage occurence. The reparation phase is always risky. Indeed, the maintenance operator can make mistakes, forget connexions or introduce undesirable elements in the product, which therefore leads to cause new damages. To account for this risk, we denote $q$ (resp. $1-q$ ) the probability for any constitutive element to be damaged (resp. to remain undamaged). The associated scheme is :

$$
\bigcirc \stackrel{q}{\rightarrow} \otimes \stackrel{1-q}{\rightarrow} \bigcirc \quad \otimes \stackrel{1}{\longrightarrow} \otimes .
$$

These assumptions of heterogeneous damages caused to a complex system come from [2] : the cell and its inward targets are herein replaced by the manufactured product and its constitutive elements respectively.

The parameters $q$ and $m$ are considered as fixed and only depend on the own feature of the product. However $r$ is allowed to vary freely : an active maintenance strategy consists in carefully repairing the failed elements so that after a few repairing phases, they will be without any default.

1.3 Let $Z_{k}$ the random variable denoting the number of safe elements in the manufactured product at time $k$. We suppose that $\left(Z_{k}\right)$ is a discrete-time Markov chain, i.e. the unit state at time $k+1$ only depends on the current state at time $k$.

Let $\boldsymbol{\Pi}$ be the corresponding transition matrix of $\left(Z_{k}\right)$. We briefly define $\boldsymbol{\Pi}$, interested readers can refer to [2] for details. The dynamics of $\left(Z_{k}\right)$ takes into account first repair mechanisms and second the phase of testing as follows :

$$
\Pi=\mathbf{R Q}
$$

where $\mathbf{R}$ models repairing and $\mathbf{Q}$ describes the procedure of tests. According to our assumptions, the matrices $\mathbf{R}$ and $\mathbf{Q}$ take the form :

$$
\begin{aligned}
& \mathbf{R}(i, j)= \begin{cases}\left(\begin{array}{c}
m-i \\
j-i
\end{array}\right) r^{j-i}(1-r)^{m-j} & i \leq j \\
0 & j<i .\end{cases} \\
& \mathbf{Q}(i, j)= \begin{cases}\left(\begin{array}{c}
i \\
j
\end{array}\right) q^{i-j}(1-q)^{j} & j \leq i<m \\
0 & i<j .\end{cases}
\end{aligned}
$$

When $i=m$,

$$
\mathbf{Q}(m, m)=1, \quad \mathbf{Q}(m, j)=0, \text { for any } 0 \leq j<m .
$$

If we assume that the product is initially in state $i_{0}\left(i_{0}\right.$ active component(s)), i.e. $P\left(Z_{0}=i_{0}\right)=1$, then

$$
P\left(Z_{k}=i\right)=\Pi^{k}\left(i_{0}, i\right) \quad i \in\{0, \ldots, m\} .
$$

The first question which is addressed herein is the efficiency measure of the repairing procedure at the element scale. Suppose that $k$ is fixed and $Z_{0}=i_{0}$. According to $(1.6), \Pi^{k}\left(i_{0}, m\right)$ is the probability that all the $m$ elements are safe after $k$ periods of time. It is clear that the design of repairing procedure is successful if $\boldsymbol{\Pi}^{k}\left(i_{0}, m\right)$ is close to 1 . The strategy is the following : choose $r$ near 1 so that $\Pi^{k}\left(i_{0}, m\right) \approx 1$. Note that if $r=1$ (all the repaired elements are safe) then $Z_{k}=m$ and $\Pi^{k}\left(i_{0}, m\right)=1$. We provide a qualitative result (see Theorem 2.1 below), which gives the exact rate of convergence of $1-\mathbf{\Pi}^{k}\left(i_{0}, m\right)$ in terms of $1-r$. This actually means that if the repairing procedure is efficient, i.e. $1-r \approx 0$, then with a high level of confidence, the $m$ elements have no default after $k$ periods. Theorem 2.1 has an interesting interpretation initially developed in a biological setting and more precisely for anti-cancer treatments [3]. Let us introduce:

$$
T:=\inf \left\{i, Z_{i}=m\right\} .
$$

Since $m$ is an absorbing state for $\left(Z_{k}\right)$,

$$
\boldsymbol{\Pi}^{k}\left(i_{0}, m\right)=P(T \leq k) .
$$


Note that $T$ is the minimal random number of periods necessary to repair and deliver a product without any default. As a result, for $r$ sufficiently close to 1 , the probability that the product is completely repaired before $k$ periods is near 1 .

The parameter $E\left(m-Z_{k}\right)$ is a second pertinent qualitative indicator evaluating the quality of the procedure. Indeed, since $Z_{k}$ converges in distribution as $r \rightarrow 1$ then $\lim _{r \rightarrow 1} E\left(Z_{k}\right)=m$. Using Theorem 2.1, the rate of convergence of $E\left(m-Z_{k}\right)$ in terms of $1-r \rightarrow 0$ is given in Proposition 2.3.

1.4 Our second goal is to measure the efficiency of the repairing procedure applied to the production of a large number $n$ of elements. We propose to take into account the performance of the whole production with the following control probability:

$$
C P_{k, n}:=P(\text { all the } n \text { products have no default at time } k) \text {. }
$$

Suppose that the repairing and test phases are independent for any product and $i_{0}$ is the common initial state of each product, then

$$
C P_{k, n}=\left(P\left(Z_{k}=m\right)\right)^{n}=\left(\Pi^{k}\left(i_{0}, m\right)\right)^{n} .
$$

It is clear that $C P_{k, n} \approx 1$ actually means that the ability of the system to repair defective products is good.

In the setting of cancer cells exposed to a treatment of radiotherapy, the analog of the parameter $C P_{k, n}$ is the tumor control probability (TCP), see [3], [2] and Section 3.

Suppose that $q$ and $k$ are fixed. The number $n$ of products being large, $C P_{k, n}$ is close to zero. This is obviously a non desirable answer. To solve this issue, we propose to take $r$ as an explicit function of $n$ such that $r \approx 1$ and the $C P_{k, n}$ is larger that a given threshold (for instance 95\%). This goal is easy to perform (see Theorem 2.5), it is a direct consequence of Theorem 2.1.

Obviously, the requirement that all the $n$ products are ready after $k$ periods, is a very stringent condition. It seems interesting to define a weaker criterium based on the proportion of products presenting a default at time $k$ :

$$
C P_{k, n, \alpha}^{*}:=P\left(\frac{N_{k}}{n} \leq \alpha\right)
$$

where $\alpha \in\left[0,1\left[\right.\right.$ and $N_{k}$ denotes the number of non-damaged products at time $k$.

It is easily seen that $N_{k}=0$ means all the $n$ elements have non-default. Consequently, $C P_{k, n} \leq C P_{k, n, \alpha}^{*}$ for any $\alpha$ and $C P_{k, n, \alpha}^{*}=C P_{k, n}$ when $\alpha=0$. We prove in Theorem 2.8 that it is actually possible to tune the parameters $r$ and $k$ such that probability $C P_{k, n, \alpha}^{*} \approx 1$.

The proposed index $C P_{k, n}$ and $C P_{k, n, \alpha}^{*}$ which measure the quality of the repairing procedure have to be compared. Suppose that $\theta_{0}$ is a given level close to 1 , for instance $\theta_{0}=95 \%$. If $1-r$ is chosen as a decreasing function of $n$ of the type $\frac{C}{n^{1 / k}}$ where $C$ is a constant, then $C P_{k, n} \approx \theta_{0}$. As for the second index, the answer is very different. Suppose that $\alpha$ is small. It can be shown that if $n$ is larger than $n_{1}$ (which only depends on $\alpha$ and $\theta_{0}$ ) and $1-r$ is proportional to $\alpha^{1 / k}$, then $C P_{k, n, \alpha}^{*} \approx \theta_{0}$. In particular, it is not necessary that $r$ depends on $n$. A more complete result can be found in Theorem 2.8 and Proposition 2.9 below.

As for the organization of the paper, the mathematical results are given in Section 2. In Section 3 we interpret our results presented in terms of reliability associated with the treatment of cancer cells by radiations. Finally the proofs of Theorems and Propositions are postponed in Section 4.

\section{The main results}

2.1 Our first goal is to study the behavior of $r \mapsto \boldsymbol{\Pi}^{k}\left(i_{0}, m\right)$ when $r \rightarrow 1$. It is intuitive that more carefully a technician (or a machine) repairs the elements (i.e. more $r$ is close to 1), more the probability of having $m$ non-damaged elements after the test phase is high. It is clear from definitions that if 
$r=1$ then $Z_{k}=m$ for any $k \geq 1$ and $r \mapsto \boldsymbol{\Pi}^{k}\left(i_{0}, m\right)$ is continuous. Note that it has been proved in [3] that $r \mapsto \Pi^{k}\left(i_{0}, m\right)$ is increasing. Therefore $Z_{k}$ converges in distribution to $m$ as $r \rightarrow 1$. The issue is to measure this convergence. It is actually possible determining the rate of decay of $1-\Pi^{k}\left(i_{0}, m\right)$ in terms of $1-r$. This result is important since it will be applied to prove Proposition 2.3 and Theorem 2.5 .

Theorem 2.1 Let $0 \leq i_{0}<m$. Set $\varepsilon:=1-r$. Then

$$
1-\Pi^{k}\left(i_{0}, m\right) \sim\left(m-i_{0}\right)(1+(m-1) q)^{k-1} \varepsilon^{k}, \quad \varepsilon \rightarrow 0 .
$$

Remark 2.2 1. Using definition (1.4) of the matrix $\mathbf{R}$, it can be proved easily (see Lemma 4.2) that $\mathbf{R}$ admits the following asymptotic expansion:

$$
\mathbf{R}=\mathbf{R}_{0}+\sum_{l=1}^{m} \mathbf{R}_{l} \epsilon^{l}, \quad(\epsilon \rightarrow 0) .
$$

Since $\mathbf{\Pi}=\mathbf{R Q}$ we have :

$$
\boldsymbol{\Pi}=\boldsymbol{\Pi}_{0}+\sum_{l=1}^{m} \Pi_{l} \epsilon^{l}, \quad(\epsilon \rightarrow 0)
$$

where $\boldsymbol{\Pi}_{l}=\mathbf{R}_{l} \mathbf{Q}$.

Therefore it is expected that $\Pi^{k}\left(i_{0}, m\right)=1+a \epsilon+\epsilon o(\epsilon)$, where $\lim _{\epsilon \rightarrow 0} o(\epsilon)=0$. Theorem 2.1 says there are non-trivial cancelations.

2. One interesting property in (2.1) is the fact that the coefficient in front of $\epsilon^{k}$ is explicitly given in terms of $i_{0}, m, q$ and $k$. This permits interpretations, see item below.

3. In [3], it has been observed that $i \mapsto \Pi^{k}(i, m)$ and $r \mapsto \Pi^{k}\left(i_{0}, m\right)$ are increasing and $q \mapsto$ $\Pi^{k}\left(i_{0}, m\right)$ is decreasing. Note that the coefficient $\varphi(i, r, q):=-(m-i)(1+(m-1) q)^{k-1}(1-r)^{k}$ inherits the same properties : $i \mapsto \varphi(i, r, q)$ and $r \mapsto \varphi(i, r, q)$ are increasing and $q \mapsto \varphi(i, r, q)$ is decreasing. These properties corresponds to intuition.

Since $Z_{k}$ converges in distribution to $m$, as $r$ goes to 1 and $Z_{k}$ takes its values in $\{0,1, \cdots, m\}$, then $\lim _{r \rightarrow 1} E\left(Z_{k}\right)=m$. Theorem 2.1 permits to give the exact rate of convergence of $m-E\left(Z_{k}\right)$.

Proposition 2.3 Suppose that $Z_{0}=i_{0}$ and $k \geq 1$. Then

$$
m-E\left(Z_{k}\right) \sim\left(m-i_{0}\right)(1+(m-1) q)^{k} \varepsilon^{k}, \quad \varepsilon \rightarrow 0 .
$$

Remark 2.4 It is interesting to introduce the time $T_{r}$ devoted to repair one element. It seems reasonable to suppose that $r$ is increasing function of $T_{r}$, for instance :

$$
r:=1-\frac{\alpha}{T_{r}^{\beta}}
$$

where $\alpha, \beta>0$.

Assume that the cost generated by repairing one element is proportional to $T_{r}$. Therefore the mean cost for repairing at time $k$ all the damaged elements is

$$
E\left(\left(m-Z_{k}\right) \gamma T_{r}\right)=\gamma T_{r}\left(m-E\left(Z_{k}\right)\right), \quad(\text { where } \gamma>0) .
$$

Therefore, under (2.4), Proposition 2.3 implies that the above quantity is closed to zero if $T_{r}$ is large and $k \beta>1$. In practice, as soon as $\beta$ is known, we have an effective way to choose $k$. 
2.2 We now consider the production of $n$ elements. We suppose that before to be delivered each element produced by the factory is repaired and tested as explained in details in Section 1.2 of Introduction. First we consider the index $C P_{k, n}$ defined by (1.10). It measures the global capability to produce non-defective elements after $k$ repair-test phases. More $C P_{k, n}$ is a close to 1 , more the maintenance may be considered as successful. To reach this objective, the parameter $r$ is taken as an increasing function of $n$ such that $C P_{k, n} \approx 1$. One second main result of this study is the following.

Theorem 2.5 Let $\left.\theta_{0} \in\right] 0,1[$. Let $r$ such that

$$
1-r:=\left(\frac{-\ln \left(\theta_{0}\right)}{\left(m-i_{0}\right)(1+(m-1) q)^{k-1}}\right)^{1 / k} \frac{1}{n^{1 / k}}
$$

Then $\lim _{n \rightarrow \infty} C P_{k, n}=\theta_{0}$.

Theorem 2.5 is a direct consequence of Theorem 2.1 .

Remark 2.6 1. In practice $\theta_{0}$ is often chosen to be equal to $95 \%$.

2. Let us give a practical consequence of Theorem 2.5. Following the analysis developed in Remark 2.4, the mean cost of the quality control applied to the $n$ elements is proportional to $\rho:=$ $n\left(m-E\left(Z_{k}\right)\right)$. Using (2.5) and Proposition 2.3, we have

$$
\begin{aligned}
\rho & \approx n\left(m-i_{0}\right)(1+(m-1) q)^{k}(1-r)^{k} \\
& \approx-\ln \left(\theta_{0}\right)(1+(m-1) q) .
\end{aligned}
$$

Consequently $\rho$ does not depend on $n$. Moreover, as expected, the function $\theta_{0} \mapsto \rho$ (resp. the functions $m \mapsto \rho$ and $q \mapsto \rho$ ) is (resp. are) decreasing (resp. increasing).

3. In the spirit of Remark 2.2, let us introduce :

$$
\Psi\left(i, q, n, k, \theta_{0}\right):=-\left(\frac{-\ln \left(\theta_{0}\right)}{(m-i)(1+(m-1) q)^{k-1}}\right)^{1 / k} \frac{1}{n^{1 / k}}
$$

It is immediate to see that $\Psi\left(i, q, n, k, \theta_{0}\right)$ is an increasing (resp. decreasing) function of either $q$ or $n$ (resp. i). Moreover it is decreasing (resp. increasing) with respect to $k$ if $n$ is large i.e. $n>\frac{-\ln \left(\theta_{0}\right)(1+(m-1) q)}{m-i}$ (resp. otherwise). It is clear that $r$ inherits the same behavior and its variations correspond to what is expected.

We now study the second parameter $C P_{k, n, \alpha}^{*}$ introduced in subsection 1.4 of the Introduction. Recall that $C P_{k, n, \alpha}^{*}$ measures efficiency of the whole system and $C P_{k, n, \alpha}^{*}$ near 1 means that the system operates successfully. To get $C P_{k, n, \alpha}^{*} \approx 1$ or equivalently $1-C P_{k, n, \alpha}^{*} \approx 0$, first we determine in Proposition 2.7 below an upper bound of $1-C P_{k, n, \alpha}^{*}$ in terms of $1-\boldsymbol{\Pi}^{k}\left(i_{0}, m\right)$. Second combining this result with Theorem 2.1 permits to prove that, under some conditions, the parameter $C P_{k, n, \alpha}^{*}$ is near 1 (see Theorem 2.8 below).

Proposition 2.7 Let $0 \leq i_{0}<m$ and $\left.\alpha \in\right] 0,1\left[\right.$. Suppose that $\boldsymbol{\Pi}^{k}\left(i_{0}, m\right)>1-\alpha$. Then

$$
1-C P_{k, n, \alpha}^{*} \leq\left\{\left(\frac{1-\boldsymbol{\Pi}^{k}\left(i_{0}, m\right)}{\alpha}\right)^{\alpha}\left(\frac{\boldsymbol{\Pi}^{k}\left(i_{0}, m\right)}{1-\alpha}\right)^{1-\alpha}\right\}^{n}, \quad \forall n \geq 1
$$

Recall that we have already observed that if $r \approx 1$, then $\boldsymbol{\Pi}^{k}\left(i_{0}, m\right)$ is close to 1 . Therefore if $r$ is in the neighborhood of 1 , then inequality (2.6) implies $C P_{k, n, \alpha}^{*} \approx 1$. 
Theorem 2.8 Let $0 \leq i_{0}<m, \alpha$ and $\left.\theta_{0} \in\right] 0,1[$. Suppose

$$
1-\left(1-\theta_{0}\right)^{1 / n} \leq \frac{\alpha(1-\alpha)^{\alpha}}{2} .
$$

1. If we have

$$
1-\Pi^{k}\left(i_{0}, m\right) \leq \alpha-\sqrt{\frac{2 \alpha\left[1-\left(1-\theta_{0}\right)^{1 / n}\right]}{(1-\alpha)^{\alpha}}}
$$

then $C P_{k, n, \alpha}^{*} \geq \theta_{0}$.

2. Suppose that $\alpha \approx 0$. If $r$ is taken so that

$$
1-r \simeq\left\{\frac{1}{\left(m-i_{0}\right)(1+(m-1) q)^{k-1}}\left(\alpha-\sqrt{\frac{2 \alpha\left[1-\left(1-\theta_{0}\right)^{1 / n}\right]}{(1-\alpha)^{\alpha}}}\right)\right\}^{1 / k}
$$

then $C P_{k, n, \alpha}^{*} \succeq \theta_{0}$.

Let us give a sufficient and easy condition under which relation (2.7) and (2.9) holds resp. Before enouncing the result let us introduce a useful notation :

$$
n_{0}:=1+\left\lfloor-\frac{2 \ln \left(1-\theta_{0}\right)}{\alpha(1-\alpha)^{\alpha}}\right\rfloor
$$

where $\lfloor a\rfloor$ stands for the integer part of $a$.

Proposition 2.9 Let $0 \leq i_{0}<m$, $\alpha$ and $\left.\theta_{0} \in\right] 0,1[$.

1. If $n \geq n_{0}$, then condition (2.7) is satisfied.

2. Suppose that $\alpha \approx 0$ and $n \geq 4 n_{0}$ and $r$ is given as

$$
1-r=\left\{\frac{\alpha}{2\left(m-i_{0}\right)(1+(m-1) q)^{k-1}}\right\}^{1 / k} .
$$

then $C P_{k, n, \alpha}^{*} \geq \theta_{0}$.

Remark 2.10 We would like to compare the two methods leading to $C P_{k, n}$ and $C P_{k, n, \alpha}^{*}$ close to $\theta_{0}$ respectively. As for the first one, Theorem 2.5 says that if $1-r$ is a decreasing function of $n$ (namely (2.5) holds) then the goal is achieved, i.e. $C P_{k, n} \approx \theta_{0}$. We emphasize that the objective $C P_{k, n, \alpha}^{*} \approx \theta_{0}$ may be obtained under a weaker condition. This result is expected since $C P_{k, n, \alpha}^{*}$ is bigger than $C P_{k, n}$. Suppose that assumptions of Proposition 2.9 are satisfied. Relation (2.11) tells us that it is not necessary to take $r$ depending on $n$ (as for the previous case), it suffices that $1-r$ is proportional to $\alpha^{1 / k}$. That shows that for $n$ large this condition is weaker than the first one.

\section{Interpretation in terms of cancer cells and tumor}

3.1 The goal of this Section is to interpret the main results given in Section 2 in the setting of an anticancer treatment by radiotherapy. The usual treatment planning in radiotherapy consists in applying a radiation dose fraction everyday $k$ to the tumor. Let us begin by briefly recalling the model introduced in [2] to take into account effects of the treatment on a cell and a tumor. At the level of a cancer cell, it is supposed :

1. a cell has $m$ vital targets. 
2. Each target may be made inactive after the application of a fraction dose of radiation with a probability $q_{0}$.

3. The cell death occurs when all the $m$ targets are deactivated.

4. Between two consecutive doses, if the cell is still alive, then an inactive target can be repaired with probability $r_{0}$. All the targets behave independently.

The aim of radiotherapy is to deliver enough radiation to the tumor to control it without irradiating normal tissue to a dose leading to serious complications (morbidity). Since radiation delivery at a precise point of cells is generally described as a random variable, the effects of the radioactive treatments on cancer and healthy cells are characterized by two probabilities: (i) the tumor Control Probability $(T C P)$ and (ii) the Normal Tissue Complication Probability $(N T C P)[16,6,9]$. TC $P_{k}$ is defined as the probability that no cancer cell remains in a tumor after applying $k$ dose fractions of radiation. $N V C P_{k, v}$, is defined as the probability that a complication appears in a normal tissue. Its expression depends on the tissue architecture. Niemierko and Goitein [12] proposed a division of normal tissues into three different architectures: serial architecture (e.g. nerves or spinal cord), parallel architecture (e.g. kidney, liver or lung) and graduated response (e.g. skin or mucous membranes). The optimum choice of radiation dose delivery technique in the treatment of a given tumor has to maximize the TCP so that at the same time the NTCP must be lower than an acceptable level (see for details [3]). In clinical radiotherapy a typical choice is $T C P \geq 0.5$ and $N T C P \leq 0.05$ [15].

Let $Z_{k}$ be the (random) number of damaged target(s) in a cell at day $k$, i.e. after application of $k$ fraction dose. It is clear that this model is the analog of the one given in the Introduction and related to reliability of engineering systems, with

$$
r:=q_{0}, \quad q:=r_{0}
$$

In practice, the probability $q_{0}$ should be an increasing function of the applied fraction dose $u_{0}$. In the linear-quadratric model of Target Theory (see Section 2.3 in [2] for details) it is supposed that

$$
q_{0}=\left(1-e^{-\gamma u_{0}-\delta u_{0}^{2}}\right)^{1 / m}
$$

where $\gamma>0$ and $\delta \geq 0$ are given parameters scaled to be adapted to the range of the possible values of $u_{0}$. In particular if $u_{0}=0$, then $q_{0}=0$, this actually means that there is no effect coming from radiation. However, as for contrary effect, it is important to note that if $u_{0}$ is large then $q_{0}$ is close to 1. In other words, there exists a natural way to force $q_{0}$ to be near 1 .

3.2 A tumor is a population of $n$ cells. We suppose that all the cells have the same independent behavior given above. In particular, the parameters $q_{0}$ and $r_{0}$ are common for all the cancer cells. The efficiency of the treatment applied to a tumor is measured by the Tumor Control Probability. This quantity is the probability that all the cancer cells are killed after application of $k$ radiation dose fractions. Due to previous assumptions of independent behaviors of constitutive cells of the tumor and (1.9)

$$
T C P_{k, n}:=C P_{k, n}=\left(P\left(Z_{k}=m\right)\right)^{m}
$$

where $C P_{k, n}$ is defined with parameters $q$ and $r$ given by (3.1).

Suppose that the tumor has a large number $n$ of cancer cells and the parameters $q_{0}$ and $r_{0}$ are fixed. Then, relation (3.3) implies that $T C P_{k, n}$ goes to 0 since $P\left(Z_{k}=m\right)<1$ and $n$ is large. This obviously means that the treatment is not efficient at all. One way to remove this undesirable conclusion is to strengthen the radiation, i.e. to choose $u_{0}$ large so that $q$ given by $(3.2)$ is close to 1 . This corresponds exactly to the problematic introduced in the above subsection 1.4. As a result, the approach developed in Section 2 may be applied.

Following subsection 1.4, let us introduce the second interesting parameter which also permits to measure efficiency of the treatment :

$$
T C P_{k, n, \alpha}^{*}:=C P_{k, n, \alpha}^{*}=P\left(\frac{N_{t}}{n} \leq \alpha\right)
$$


where $\frac{N_{t}}{n}$ is the fraction of malign cells which have not been destroyed by the treatment.

Recall that $T C P_{k, n} \leq T C P_{k, n}^{*}$ and the goal is to have either $T C P_{k, n}$ or $T C P_{k, n}^{*}$ close to 1 . Here, this objective can be achieved choosing $q_{0} \approx 1$ or equivalently $u_{0}$ large.

3.3 It is interesting to interpret the main results given in Section 2 and their consequences on the radiotherapy response model. We keep notation given in Section 2.

\section{Interpretation of Proposition 2.3 and Remark 2.4}

Relation (2.3) can be written as :

$$
m-E\left(Z_{k}\right) \sim\left(m-i_{0}\right)\left(1+(m-1) r_{0}\right)^{k}\left(1-q_{0}\right)^{k}, \quad q_{0} \rightarrow 1 .
$$

where $i_{0} \in\{0,1, \cdots, m-1\}$ is the initial state of the cell, $Z_{k}$ its state at time $k$.

Using moreover (3.2) we get

$$
m-E\left(Z_{k}\right) \sim\left(m-i_{0}\right)\left(1+(m-1) r_{0}\right)^{k}\left[1-\left(1-e^{-\gamma u_{0}-\delta u_{0}^{2}}\right)^{1 / m}\right]^{k}, \quad u_{0} \rightarrow \infty .
$$

In particular relation (3.6) provides a quantitative way to choose the dose fraction $u_{0}$ large so that the mean of $Z_{k}$ is close to 1 .

\section{Interpretation of Theorem 2.5 and Remark 2.6}

$T C P_{k, n}$ converges to a given real number $\theta_{0}$ as soon as

$$
1-q_{0}:=\left(\frac{-\ln \left(\theta_{0}\right)}{\left(m-i_{0}\right)\left(1+(m-1) r_{0}\right)^{k-1}}\right)^{1 / k} \frac{1}{n^{1 / k}} .
$$

This formula can be applied in practice once we have an estimate of the number of cancer cells. Indeed, recall that $q_{0}$ is expressed via the dose fraction $u_{0}$ by (3.2). Therefore $u_{0}$ and $k$ can be determined so that the treatment has a good chance to be successful, i.e. the probability to kill the whole tumor equals $.95 \%$ for instance.

The comments related to the variation of the coefficient $\psi\left(i, r_{0}, n, k, \theta_{0}\right)$ given in item 3 of Remark 2.6 remain valid in the biological context.

\section{Interpretation of Theorem 2.8 and Proposition 2.9}

We now focus on the second indicator $T C P_{k, n, \alpha}^{*}$ and the goal is again to force $T C P_{k, n, \alpha}^{*} \approx 1$. Let us start with a small $\alpha$, for instance $\alpha=.001$. Suppose that the size of the tumor before the beginning of the treatment is important. If $u_{0}$ is chosen such that

$$
\gamma u_{0}+\delta u_{0}^{2}=-\ln \left(1-\left[1-\left\{\frac{\alpha}{2\left(m-i_{0}\right)\left(1+(m-1) r_{0}\right)^{k-1}}\right\}^{1 / k}\right]^{m}\right)
$$

then $T C P_{k, n, \alpha}^{*} \approx \theta_{0}$.

Introducing

$$
\psi_{0}:=\frac{1}{2\left(m-i_{0}\right)\left(1+(m-1) r_{0}\right)^{k-1}} .
$$

and using

$$
\left[1-\left\{\frac{\alpha}{\psi_{0}}\right\}^{1 / k}\right]^{m}=1-\frac{m}{\psi_{0}^{1 / k}} \alpha^{1 / k}+o\left(\alpha^{1 / k}\right), \quad \alpha \rightarrow 0
$$

we finally obtain :

$$
\gamma u_{0}+\delta u_{0}^{2} \approx-\frac{1}{k} \ln (\alpha)
$$

Note that $u_{0}$ implicitly depends on $\theta_{0}$ and $\alpha$ since it is supposed that the initial number of cancer cells constituting the tumor is larger than the threshold $n_{0}$ given by $(2.10)$.

Moreover (3.9) corresponds to intuition : the smaller $\alpha$ is, the larger $u_{0}$ has to be. 


\section{Proofs}

\subsection{Proof of Theorem 2.1}

We keep notation introduced in Sections 1 and 2.We adopt the convention that for any matrix the first line and the first column are labeled 0 . We begin with a preliminary result which will be used several times.

Lemma 4.1 Let $B_{1}$ and $B_{2}$ two square matrix $(m+1) \times(m+1)$. Consider $\mathbf{R}_{0}$ the square matrix defined as

$$
\mathbf{R}_{0}(i, m)=\left\{\begin{array}{cc}
1 & \text { for any } 0 \leq i \leq m \\
0 & \text { otherwise }
\end{array}\right.
$$

Suppose :

$$
\sum_{j=0}^{m} B_{2}(i, j)=a, \quad \forall i \in\{0,1, \cdots, m\} .
$$

Then

$$
B_{1} B_{2} \mathbf{R}_{0}(i, m)=a B_{1} \mathbf{R}_{0}(i, m), \quad \forall i \in\{0,1, \cdots, m\} .
$$

Proof. According to the definition of $\mathbf{R}_{0}$ and (4.1), we have :

$$
B_{1} B_{2} \mathbf{R}_{0}(i, m)=\sum_{j=0}^{m} B_{1} B_{2}(i, j)=\sum_{k=0}^{m} B_{1}(i, k) \sum_{j=0}^{m} B_{2}(k, j)=a \sum_{k=0}^{m} B_{1}(i, k)=B_{1} \mathbf{R}_{0}(i, m) .
$$

In Lemma 4.2 below, we give the behavior of $\mathbf{R}$ as a function of

$$
\epsilon:=1-r
$$

when $\epsilon \rightarrow 0$.

This allows us to determine the asymptotic expansion of $\boldsymbol{\Pi}$ in Lemma 4.5.

Lemma 4.2 The matrix $\mathbf{R}$ admits the following asymptotic expansion

$$
\mathbf{R}=\mathbf{R}_{0}+\sum_{l=1}^{m} \mathbf{R}_{l} \epsilon^{l}, \quad(\epsilon \rightarrow 0)
$$

where for $1 \leq l \leq m, \mathbf{R}_{l}$ is the $(m+1) \times(m+1)$ matrix

$$
\mathbf{R}_{l}(i, j):=\left(\begin{array}{c}
m-i \\
l
\end{array}\right)\left(\begin{array}{c}
l \\
m-j
\end{array}\right)(-1)^{l+j-m}
$$

if $0 \leq i \leq j, i<m$ and $m-j \leq l \leq m-i$. Otherwise $\mathbf{R}_{l}(i, j)=0$.

Proof. Using (1.4) and (4.3) we have :

$$
\mathbf{R}(i, j)=\left(\begin{array}{c}
m-i \\
j-i
\end{array}\right)(1-\epsilon)^{j-i} \epsilon^{m-j}
$$

where $0 \leq i \leq j \leq m$ and $\mathbf{R}(i, j)=0$ otherwise. 
Developing $(1-\epsilon)^{j-i}$ we get

$$
\mathbf{R}(i, j)=\sum_{r=0}^{j-i}\left(\begin{array}{l}
m-i \\
j-i
\end{array}\right)\left(\begin{array}{l}
j-i \\
r
\end{array}\right)(-1)^{r} \epsilon^{m+r-j} .
$$

Setting $l:=m+r-j$ leads to (4.4) where

$$
\mathbf{R}_{l}(i, j):=(-1)^{l+j-m}\left(\begin{array}{c}
m-i \\
j-i
\end{array}\right)\left(\begin{array}{l}
j-i \\
l+j-m
\end{array}\right) \mathbf{1}_{\{m-j \leq l \leq m-i\}} .
$$

Since :

$$
\left(\begin{array}{c}
m-i \\
j-i
\end{array}\right)\left(\begin{array}{l}
j-i \\
l+j-m
\end{array}\right)=\left(\begin{array}{c}
m-i \\
l
\end{array}\right)\left(\begin{array}{c}
l \\
m-j
\end{array}\right)
$$

the result follows.

Lemma 4.3 1. $\mathbf{R}_{0} \mathbf{Q}=\mathbf{R}_{0}$.

2. For any $1 \leq l \leq m$, we have :

$$
\mathbf{R}_{0}^{l}=\mathbf{R}_{0}, \quad \mathbf{R}_{0} \mathbf{R}_{l}=0, \quad \mathbf{R}_{0} \mathbf{R}_{l} \mathbf{Q}=0 .
$$

Proof. The proof is straightforward and is left to the reader.

Definition 4.4 A multi-index $\underline{i}$ is an element of $\bigcup_{k \geq 1}\{1,2, \cdots, m\}^{k}$. For any multi-index $\underline{i}=$ $\left(i_{1}, \cdots, i_{k}\right)$ we set :

$$
\begin{gathered}
|\underline{i}|:=k, \quad\|\underline{i}\|:=i_{1}+\cdots+i_{k}, \\
\boldsymbol{\Pi}_{\underline{i}}:=\boldsymbol{\Pi}_{i_{1}} \times \cdots \times \boldsymbol{\Pi}_{i_{k}} .
\end{gathered}
$$

where

$$
\Pi_{l}:=\mathbf{R}_{l} \mathbf{Q}
$$

Lemma 4.5 The matrix $\Pi^{k}$ admits the following asymptotic expansion :

$$
\boldsymbol{\Pi}^{k}=\mathbf{R}_{0}+\sum_{|\underline{i}|=k} \epsilon^{\|\underline{\underline{i}}\|} \boldsymbol{\Pi}_{\underline{i}}+\sum_{|\underline{i}| \leq k} \epsilon^{\|\underline{i}\|} \boldsymbol{\Pi}_{\underline{i}} \mathbf{R}_{0} .
$$

Proof. Since $\boldsymbol{\Pi}=\mathbf{R Q}$, then relation (4.4) implies

$$
\boldsymbol{\Pi}^{k}=\left(\sum_{l=0}^{m} \epsilon^{l} \boldsymbol{\Pi}_{l}\right)^{k}=\sum_{i_{1}, \cdots, i_{k}} \boldsymbol{\Pi}_{i_{1}} \times \cdots \times \boldsymbol{\Pi}_{i_{k}} \epsilon^{i_{1}+\cdots+i_{k}}
$$

where $i_{1}, \cdots, i_{k}$ belong to $\{0,1, \cdots, m\}$.

We now consider three different cases.

a) If $i_{1}=i_{2}=\cdots=i_{k}=0$ then the corresponding term is $\mathbf{R}_{0}^{k}=\mathbf{R}_{0}$.

b) If each $i_{j}$ belongs to $\{1,2, \cdots, m\}$, set $\underline{i}:=\left(i_{1}, \cdots, i_{k}\right)$. Then $|\underline{i}|=k$ and $\|\underline{i}\|=i_{1}+\cdots+i_{k} \geq k$.

This gives the second term in the right hand-side of (4.5).

c) The third and last case is the one where $\left(i_{1}, \cdots, i_{k}\right) \neq(0, \cdots, 0)$ and there exists $j$ such that $i_{j}=0$.

There are two possibilities :

1. $\left(i_{1}, i_{2}, \cdots, i_{k}\right)=\left(i_{1}, i_{2}, \cdots, i_{k^{\prime}}, i_{k^{\prime}+1}, \cdots, i_{k}\right)$ where $i_{k^{\prime}}=0$ and $i_{k^{\prime}+1} \geq 1, \cdots, i_{k} \geq 1$,

2. $\left(i_{1}, i_{2}, \cdots, i_{k}\right)=\left(i_{1}, i_{2}, \cdots, i_{k^{\prime}}, i_{k^{\prime}+1}, \cdots, i_{k}\right)$ where $i_{k^{\prime}} \geq 1$ and $i_{k^{\prime}+1}=\cdots=i_{k}=0$. 
In the first case,

$$
\Pi_{i_{1}} \times \cdots \times \boldsymbol{\Pi}_{i_{k}}=\Pi_{i_{1}} \times \cdots \times \boldsymbol{\Pi}_{i_{k^{\prime}}} \boldsymbol{\Pi}_{i_{k^{\prime}+1}} \times \cdots \times \boldsymbol{\Pi}_{i_{k}} .
$$

The above product vanishes since Lemma 4.3 implies that

$$
\boldsymbol{\Pi}_{i_{k^{\prime}}} \boldsymbol{\Pi}_{i_{k^{\prime}+1}}=\mathbf{R}_{0} \mathbf{Q} \mathbf{R}_{i_{k^{\prime}+1}} \mathbf{Q}=\mathbf{R}_{0} \mathbf{R}_{i_{k^{\prime}+1}} \mathbf{Q}=0 .
$$

As for the second case, we have :

$$
\boldsymbol{\Pi}_{i_{k^{\prime}+1}} \times \cdots \times \boldsymbol{\Pi}_{i_{k}}=\left(\mathbf{R}_{0} \mathbf{Q}\right) \times \cdots \times\left(\mathbf{R}_{0} \mathbf{Q}\right)=\mathbf{R}_{0} \times \cdots \times \mathbf{R}_{0}=\mathbf{R}_{0} .
$$

If there exists $1 \leq j<k^{\prime}$ such that $i_{j}=0$, proceeding similarly leads to $\Pi_{i_{1}} \times \cdots \times \boldsymbol{\Pi}_{i_{k^{\prime}}}=0$. Finally it can be supposed that $\left(i_{1}, \cdots, i_{k^{\prime}}\right)$ is a multi-index with length $k^{\prime}$.

Remark 4.6 Lemma 4.5 is crucial in the proof of Theorem 2.1. Indeed, we will see in Lemma 4.8 below that the terms in the second sum of the right hand-side of (4.5) do not contribute to the equivalent of $1-\Pi^{k}\left(i_{0}, m\right)$, as $\epsilon \rightarrow 0$.

Lemma 4.7 We have the following identities :

$$
\sum_{j=0}^{m} \mathbf{R}_{l}(i, j)=0, \quad \forall i \in\{0,1, \cdots, m\}, l \geq 1 .
$$

Proof. 1) If $i=m$ then according to the definition of the matrix $\mathbf{R}_{l}$, we have $\mathbf{R}_{l}(m, j)=0$ for any $0 \leq j \leq m$.

From now on suppose that $i<m$. Using Lemma 4.2 we get

$$
\sum_{j=0}^{m} \mathbf{R}_{l}(i, j)=\left(\begin{array}{c}
m-i \\
l
\end{array}\right) \sum_{j=0}^{m}\left(\begin{array}{c}
l \\
m-j
\end{array}\right)(-1)^{l+j-m} \mathbf{1}_{\{i \leq j, m-j \leq l \leq m-i\}} .
$$

Setting $s=j-m+l$ we obtain :

$$
\sum_{j=0}^{m} \mathbf{R}_{l}(i, j)=\left(\begin{array}{c}
m-i \\
l
\end{array}\right) \mathbf{1}_{\{l \leq m-i\}} \sum_{s=0}^{l}\left(\begin{array}{c}
l \\
l-s
\end{array}\right)(-1)^{s} .
$$

Since $\sum_{s=0}^{l}\left(\begin{array}{c}l \\ l-s\end{array}\right)(-1)^{s}=(1-1)^{l}=0$, then Lemma 4.7 follows.

Lemma 4.8 Let $\underline{i}$ be a multi-index, then:

$$
\boldsymbol{\Pi}_{\underline{i}} \mathbf{R}_{0}(k, m)=0, \quad \text { for any } k \in\{0,1, \cdots, m\} .
$$

Proof. We prove (4.6), reasoning by induction on the length $n$ of $\underline{i}$.

1) Let us start with $n=1$. Then $\underline{i}=i \geq 1$ and

$$
\boldsymbol{\Pi}_{\underline{i}} \mathbf{R}_{0}(k, m)=\boldsymbol{\Pi}_{i} \mathbf{R}_{0}(k, m)=\mathbf{R}_{i} \mathbf{Q} \mathbf{R}_{0}(k, m) .
$$

We claim that Lemma 4.1 may be applied.

If $i<m$, then using (1.5), we get

$$
\sum_{j=0}^{m} \mathbf{Q}(i, j)=\sum_{j=0}^{i}\left(\begin{array}{c}
i \\
j
\end{array}\right) q^{i-j}(1-q)^{j}=(1+1-q)^{i}=1 .
$$

Since $\mathbf{Q}(m, j)=\mathbf{1}_{\{m=j\}}$, it is clear that (4.7) holds when $i=m$. 
As a result we may apply Lemma 4.1 with $B_{1}:=\mathbf{R}_{i}, B_{2}=\mathbf{Q}$ and $a=1: \mathbf{R}_{i} \mathbf{Q} \mathbf{R}_{0}(k, m)=\mathbf{R}_{i} \mathbf{R}_{0}(k, m)$. Lemma 4.7 implies that we can apply twice Lemma 4.1 with $B_{1}:=\mathbf{I}, B_{2}=\mathbf{R}_{i}$ and $a=0$ : $\mathbf{R}_{i} \mathbf{R}_{0}(k, m)=0$. Consequently $\boldsymbol{\Pi}_{\underline{i}} \mathbf{R}_{0}(k, m)=0$.

2 ) It remains to prove $n \rightarrow n+1$. Let $\underline{i}$ be a multi-index of length $n+1$. It can be written as $\underline{i}=\left(j, \underline{i^{\prime}}\right)$ where $\underline{i}^{\prime}$ is a multi-index with length $n$ and $j \geq 1$. Consequently, for any $i_{0} \in\{1,2, \cdots, m\}$, we have :

$$
\boldsymbol{\Pi}_{\underline{i}} \mathbf{R}_{0}(k, m)=\boldsymbol{\Pi}_{j} \boldsymbol{\Pi}_{\underline{i^{\prime}}} \mathbf{R}_{0}(k, m)=\sum_{s=0}^{m} \boldsymbol{\Pi}_{j}(k, s)\left(\boldsymbol{\Pi}_{\underline{i^{\prime}}} \mathbf{R}_{0}\right)(s, m)=0
$$

since $\underline{i^{\prime}}$ being a multi-index with length $n$, then $\left(\boldsymbol{\Pi}_{\underline{i^{\prime}}} \mathbf{R}_{0}\right)(s, m)=0$.

Lemma 4.9 For any $0 \leq i<m$ we have

$$
\boldsymbol{\Pi}_{1}(i, k)= \begin{cases}-(m-i) & \text { if } k=m \\
(m-i)\left(\begin{array}{c}
m-1 \\
k
\end{array}\right) q^{m-1-k}(1-q)^{k}, & \text { if } 0 \leq k<m\end{cases}
$$

Moreover $\Pi_{1}^{s}(m, j)=0$ for any $0 \leq j \leq m$ and $s \geq 1$.

Proof. Suppose that $0 \leq i<m$ and $0 \leq j \leq m$. Using the definition of $\mathbf{R}_{1}$ (cf Lemma 4.2) we easily prove that $\mathbf{R}_{1}(i, j)=0$ if $j<m-1, \mathbf{R}_{1}(i, m-1)=m-i$ and $\mathbf{R}_{1}(i, m)=-(m-i)$. As a result :

$$
\boldsymbol{\Pi}_{1}(i, k)=\mathbf{R}_{1} \mathbf{Q}(i, k)=\sum_{j=0}^{m} \mathbf{R}_{1}(i, j) \mathbf{Q}(j, k)=(m-i)(\mathbf{Q}(m-1, k)-\mathbf{Q}(m, k)) .
$$

Using (1.5), we get (4.8).

It remains to study the case $i=m$. Du to the fact that $\mathbf{R}_{1}(m, j)=0$ for any $j$, we deduce :

$$
\boldsymbol{\Pi}_{1}(m, k)=\mathbf{R}_{1} \mathbf{Q}(m, k)=\sum_{j=0}^{m} \mathbf{R}_{1}(m, j) \mathbf{Q}(j, k)=0, \quad \forall k \in\{0,1, \cdots, m\} .
$$

Let $s \geq 1$ and $0 \leq j \leq m$. We have :

$$
\boldsymbol{\Pi}_{1}^{s+1}(m, j)=\sum_{i=0}^{m} \boldsymbol{\Pi}_{1}(m, i) \boldsymbol{\Pi}_{1}^{s}(i, j)=0 .
$$

Lemma 4.10 For any $0 \leq i<m$ we have

$$
\Pi_{1}^{k}(i, m)=-(m-i)(1+(m-1) q)^{k-1} .
$$

Proof. 1) When $k=1$, identity (4.9) is a direct consequence of Lemma 4.9.

Let us deal with $k=2$. Let $0 \leq i<m$. Using Lemma 4.9 we get :

$$
\boldsymbol{\Pi}_{1}^{2}(i, m)=\sum_{j=0}^{m-1} \boldsymbol{\Pi}_{1}(i, j) \boldsymbol{\Pi}_{1}(j, m)=-\sum_{j=0}^{m-1}(m-i)\left(\begin{array}{c}
m-1 \\
j
\end{array}\right) q^{m-1-j}(1-q)^{j}(m-j) .
$$

It is convenient to introduce

$$
S(x):=\sum_{j=0}^{m-1}(m-j)\left(\begin{array}{c}
m-1 \\
j
\end{array}\right) x^{m-1-j}, \quad x \in \mathbb{R} .
$$


Indeed,

$$
\boldsymbol{\Pi}_{1}^{2}(i, m)=-(m-i)(1-q)^{m-1} S\left(\frac{q}{1-q}\right)
$$

Let us calculate $S(x)$ :

$$
S(x)=\frac{d}{d x}\left(\sum_{j=0}^{m-1}\left(\begin{array}{c}
m-1 \\
j
\end{array}\right) x^{m-j}\right)=\frac{d}{d x}\left(x(1+x)^{m-1}\right)=(1+m x)(1+x)^{m-2} .
$$

We easily deduce that

$$
\boldsymbol{\Pi}_{1}^{2}(i, m)=-(m-i)(1+(m-1) q) .
$$

3) We now prove (4.9) by induction on $k$. We have already proved it for $k=1$ and $k=2$. Let us detail $k \rightarrow(k+1)$. Observe that

$$
\boldsymbol{\Pi}_{1}^{k}(j, m)=-(m-j)(1+(m-1) q)^{k-1}=(1+(m-1) q)^{k-1} \boldsymbol{\Pi}_{1}(j, m), \quad 0 \leq j<m .
$$

Let $0 \leq i<m$. We know from Lemma 4.9 that $\Pi_{1}^{k}(m, m)=0$, consequently

$$
\begin{aligned}
\boldsymbol{\Pi}_{1}^{k+1}(i, m) & =\sum_{j=0}^{m-1} \boldsymbol{\Pi}_{1}(i, j) \boldsymbol{\Pi}_{1}^{k}(j, m)=(1+(m-1) q)^{k-1} \sum_{j=0}^{m-1} \boldsymbol{\Pi}_{1}(i, j) \boldsymbol{\Pi}_{1}(j, m) \\
& =(1+(m-1) q)^{k-1} \boldsymbol{\Pi}_{1}^{2}(i, m)=-(m-i)(1+(m-1) q)^{k} .
\end{aligned}
$$

\section{Proof of Theorem 2.1}

Let $0 \leq i_{0}<m$. Recall that $\mathbf{R}_{0}\left(i_{0}, m\right)=1$, therefore Lemmas 4.5 and 4.8 imply :

$$
1-\boldsymbol{\Pi}^{k}\left(i_{0}, m\right)=-\sum_{|\underline{\mid}|=k} \epsilon^{\|\underline{i}\|} \boldsymbol{\Pi}_{\underline{i}}\left(i_{0}, m\right)-\sum_{|\underline{i}| \leq k} \epsilon^{\|\underline{i}\|} \boldsymbol{\Pi}_{\underline{i}} \mathbf{R}_{0}\left(i_{0}, m\right)=-\sum_{|\underline{i}|=k} \epsilon^{\|\underline{i}\|} \boldsymbol{\Pi}_{\underline{i}}\left(i_{0}, m\right) .
$$

It is clear that

$$
\sum_{|\underline{i}|=k} \epsilon^{\|\underline{i}\|} \boldsymbol{\Pi}_{\underline{i}}\left(i_{0}, m\right) \sim \epsilon^{\left\|\underline{j_{0}}\right\|} \boldsymbol{\Pi}_{\underline{j_{0}}}\left(i_{0}, m\right), \quad \epsilon \rightarrow 0
$$

where $\underline{j_{0}}:=(1,1, \cdots, 1)$.

Moreover Lemma 4.10 leads to

$$
1-\Pi^{k}\left(i_{0}, m\right) \sim \epsilon^{k} \Pi_{1}^{k}\left(i_{0}, m\right) \sim-\left(m-i_{0}\right)(1+(m-1) q)^{k-1} \epsilon^{k}, \quad \epsilon \rightarrow 0 .
$$

\subsection{Proof of Proposition 2.3}

First we focus on the calculation of $E\left(Z_{k}\right)$. Although it is not possible to give an explicit value of the expection of $Z_{k}$ we are however able to provide a recursive relation between $E\left(Z_{k+1}\right)$ and $E\left(Z_{k}\right)$.

Lemma 4.11 Suppose that $Z_{0}=i_{0}$ and $k \geq 0$. Then

$$
E\left(Z_{k+1}\right)=(1-q) m r+q m E\left(r^{m-Z_{k}}\right)+(1-q)(1-r) E\left(Z_{k}\right) .
$$

Proof. Denote $Z^{\prime}$ the number of non-damaged elements after the repairing procedure applied to one element. According to the scheme (1.2), conditionally on $Z^{\prime}=j<m$, the distribution of $Z_{k+1}$ is binomial with parameters $j$ and $1-q$. Therefore

$$
E\left(Z_{k+1} \mid Z^{\prime}=j\right)=(1-q) j .
$$


When $Z^{\prime}=m$, then $Z_{k+1}=m$.

Consequently :

$$
E\left(Z_{k+1}\right)=E\left(1_{\left\{Z^{\prime}=m\right\}} m\right)+E\left(1_{\left\{Z^{\prime}<m\right\}}(1-q) Z^{\prime}\right)=q m P\left(Z^{\prime}=m\right)+(1-q) E\left(Z^{\prime}\right) .
$$

We deduce from (1.1) that conditionally on $Z_{k}=i, Z^{\prime}-i \sim \mathcal{B}(m-i, r)$. That implies

$$
P\left(Z^{\prime}=m \mid Z_{k}=i\right)=r^{m-i}, \quad E\left(Z^{\prime} \mid Z_{k}=i\right)=i+(m-i) r=m r+(1-r) i
$$

and

$$
E\left(Z_{k+1}\right)=q m E\left(r^{m-Z_{k}}\right)+m r(1-q)+(1-q)(1-r) E\left(Z_{k}\right) .
$$

Proposition 2.3 will proved by induction on $k$. First we study the case $k=1$ in Lemma 4.12 below.

Lemma 4.12 Suppose that $Z_{0}=i_{0}$, then

$$
m-E\left(Z_{1}\right) \sim(1+(m-1) q)\left(m-i_{0}\right) \epsilon, \quad \epsilon \rightarrow 0
$$

where $\epsilon=1-r$.

Proof. Using Lemma 4.11 with $k=0$ we obtain :

$$
\begin{aligned}
E\left(Z_{1}\right) & =(1-q) m r+q m r^{m-i_{0}}+(1-q)(1-r) i_{0} \\
& =(1-q) m(1-\epsilon)+q m(1-\epsilon)^{m-i_{0}}+(1-q) i_{0} \epsilon
\end{aligned}
$$

Since $(1-\epsilon)^{m-i_{0}}=1-\left(m-i_{0}\right) \epsilon+o(\epsilon)$, we deduce :

$$
E\left(Z_{1}\right)=m-(1+(m-1) q)\left(m-i_{0}\right) \epsilon+o(\epsilon) .
$$

\section{Proof of Proposition 2.3}

Let us prove by induction on $k$ :

$$
m-E\left(Z_{k}\right) \sim\left(m-i_{0}\right)(1+(m-1) q)^{k} \varepsilon^{k}, \quad \varepsilon \rightarrow 0 .
$$

Obviously the case $k=1$ corresponds to Lemma 4.12. Suppose that (4.11) holds and prove

$$
m-E\left(Z_{k+1}\right) \sim\left(m-i_{0}\right)(1+(m-1) q)^{k+1} \varepsilon^{k+1}, \quad \varepsilon \rightarrow 0 .
$$

Using Lemma 4.11 and (4.11) we get :

$$
\begin{aligned}
E\left(Z_{k+1}\right)= & (1-q) m(1-\epsilon)+q m E\left((1-\epsilon)^{m-Z_{k}}\right)+(1-q) \epsilon E\left(Z_{k}\right) \\
= & (1-q) m(1-\epsilon)+q m E\left(1-\left(m-Z_{k}\right) \epsilon\right)+q m R(\epsilon) \\
& +(1-q) \epsilon\left[m-\left(m-i_{0}\right)(1+(m-1) q)^{k} \varepsilon^{k}\right]+o\left(\varepsilon^{k}\right)
\end{aligned}
$$

where

$$
R(\epsilon):=E\left((1-\epsilon)^{m-Z_{k}}-\left[1-\left(m-Z_{k}\right) \epsilon\right]\right) .
$$

Starting with the classical inequality :

$$
\left.\left|(1-\epsilon)^{n}-(1-n \epsilon)\right| \leq \frac{n(n-1)}{2} \epsilon^{2}, \quad \forall \epsilon \in\right] 0,1[
$$


we deduce

$$
\left.\left|(1-\epsilon)^{n}-(1-n \epsilon)\right| \leq \frac{m-1}{2} n \epsilon^{2}, \quad 0 \leq n \leq m, \epsilon \in\right] 0,1[.
$$

Consequently,

$$
|R(\epsilon)| \leq \frac{m-1}{2} E\left(m-Z_{k}\right) \epsilon^{2} .
$$

Using (4.11) we conclude that $R(\epsilon)=o\left(\epsilon^{k+1}\right)$ and

$$
\begin{aligned}
E\left(Z_{k+1}\right)= & m-(1-q) m \epsilon-q m \epsilon\left(m-i_{0}\right)(1+(m-1) q)^{k} \varepsilon^{k} \\
& +(1-q) \epsilon\left[m-\left(m-i_{0}\right)(1+(m-1) q)^{k} \varepsilon^{k}\right]+o\left(\varepsilon^{k+1}\right) \\
= & m-\left(m-i_{0}\right)(1+(m-1) q)^{k}(q m+1-q) \epsilon^{k+1}+o\left(\varepsilon^{k+1}\right) \\
= & m-\left(m-i_{0}\right)(1+(m-1) q)^{k+1} \epsilon^{k+1}+o\left(\varepsilon^{k+1}\right) .
\end{aligned}
$$

This implies (4.12).

\subsection{Proof of Theorem 2.5}

Let $0 \leq i_{0}<m$ and $k \geq 1$. Suppose that identity (2.5) holds. Therefore $r$ is a function of $n$ and moreover $\epsilon:=1-r$ goes to 0 as $n \rightarrow \infty$. According to the definition (1.10) of $C P_{k, n}$, we have :

$$
\ln \left(C P_{k, n}\right)=n \ln \left(\boldsymbol{\Pi}^{k}\left(i_{0}, m\right)\right)=n \ln \left(1-\left[1-\boldsymbol{\Pi}^{k}\left(i_{0}, m\right)\right]\right) .
$$

Theorem 2.1 and (2.5) imply that :

$$
\ln \left(C P_{k, n}\right) \sim-n\left[1-\boldsymbol{\Pi}^{k}\left(i_{0}, m\right)\right] \sim-n\left(m-i_{0}\right)(1+(m-1) q)^{k-1} \epsilon^{k} \sim \ln \left(\theta_{0}\right), \quad n \rightarrow \infty .
$$

\subsection{Proof of Proposition 2.7}

Let $\left(\left(Z_{k}^{(i)}\right)\right)_{i \geq 1}$ a collection of independent Markov chains with common transition matrix $\boldsymbol{\Pi}$ given by (1.3) and having the same initial point $i_{0}$. Let us introduce :

$$
N_{k}=\sum_{i=1}^{n} \xi_{i}, \quad \text { where } \xi_{i}:=\mathbf{1}_{\left\{Z_{k}^{(i)} \neq m\right\}}
$$

Recall that from (1.11) we have :

$$
1-C P_{k, n, \alpha}^{*}:=P\left(\frac{N_{k}}{n}>\alpha\right) .
$$

The technique developed below is classical in the theory of large deviations. In our specific setting (2.6) is a particular case of Cramér's theorem (see Section 2.2 in [7] and more specifically Exercise $2.2 .23)$.

Note that $\left\{\frac{N_{k}}{n}>\alpha\right\}=\left\{\exp \left\{\lambda \sum_{i=1}^{n} \xi_{i}\right\}>e^{\lambda \alpha n}\right\}$ for any $\lambda>0$. Using the Markov inequality and the fact that r.v.'s $\xi_{i}$ are iid, we get :

$$
1-C P_{k, n, \alpha}^{*} \leq e^{-\lambda \alpha n} E\left(\exp \left\{\lambda \sum_{i=1}^{n} \xi_{i}\right\}\right)=\left(e^{-\lambda \alpha} E\left(e^{\lambda \xi_{1}}\right)\right)^{n}=e^{n a(\lambda)}
$$

where

$$
a(\lambda):=-\lambda \alpha+\ln \left(E\left(e^{\lambda \xi_{1}}\right)\right)=-\lambda \alpha+\ln \left(p+(1-p) e^{\lambda}\right)
$$


and $p:=P\left(\xi_{1}=0\right)=P\left(Z_{k}^{(1)}=m\right)=\Pi^{k}\left(i_{0}, m\right)$.

Using classical analysis it can be proved that $a(\lambda) \geq a\left(\lambda^{*}\right)$ for any $\lambda>0$ where

$$
\lambda^{*}:=\ln \left(\frac{p \alpha}{(1-p)(1-\alpha)}\right) .
$$

Observe that $\lambda^{*}>0$ since $1-\alpha<p$. Moreover

$$
a\left(\lambda^{*}\right)=-\alpha \ln \left(\frac{p \alpha}{(1-p)(1-\alpha)}\right)+\ln \left(\frac{p}{1-\alpha}\right)=\ln \left(\left(\frac{1-p}{\alpha}\right)^{\alpha}\left(\frac{p}{1-\alpha}\right)^{1-\alpha}\right) .
$$

It is clear that (2.6) is a direct consequence of (4.13) (with $\lambda=\lambda^{*}$ ) and (4.15).

\subsection{Proof of Theorem 2.8}

Let $\left.\theta_{0} \in\right] 0,1[$ and $\alpha$ in $] 0,1[$. Let us introduce the function :

$$
f(x):=\left(\frac{1-x}{\alpha}\right)^{\alpha}\left(\frac{x}{1-\alpha}\right)^{1-\alpha}, \quad 1-\alpha \leq x \leq 1
$$

and

$$
g(y):=f(1-\alpha+\alpha y), \quad \forall y \in[0,1] .
$$

Obviously,

$$
g(y)=(1-y)^{\alpha}\left(1+\frac{\alpha}{1-\alpha} y\right)^{1-\alpha}, \quad 0 \leq y \leq 1 .
$$

Lemma 4.13 The function $g$ is concave over $[0,1]$ and

$$
g(y) \leq 1-\frac{\alpha(1-\alpha)^{\alpha}}{2} y^{2}, \quad \forall y \in[0,1] .
$$

Proof. 1) Taking the second derivative, we get :

$$
g^{\prime \prime}(y)=(1-y)^{\alpha-2}\left(1+\frac{\alpha}{1-\alpha} y\right)^{-\alpha-1} h(y)
$$

where

$$
\begin{aligned}
h(y)= & \alpha(\alpha-1)\left(1+\frac{\alpha}{1-\alpha} y\right)^{2}-2 \alpha(1-\alpha) \frac{\alpha}{1-\alpha}(1-y)\left(1+\frac{\alpha}{1-\alpha} y\right) \\
& -(1-\alpha) \alpha \frac{\alpha^{2}}{(1-\alpha)^{2}}(1-y)^{2} \\
= & \alpha\left[(\alpha-1)\left(1+\frac{\alpha}{1-\alpha} y\right)^{2}-2 \alpha(1-y)\left(1+\frac{\alpha}{1-\alpha} y\right)-\frac{\alpha^{2}}{1-\alpha}(1-y)^{2}\right] \\
= & -\frac{\alpha}{1-\alpha} .
\end{aligned}
$$

This identity implies that $g^{\prime \prime}(y)<0$ for all $y$ in $] 0,1[$ and therefore $g$ is concave over $[0,1]$. 2) Since

$$
g^{\prime}(y)=(1-y)^{\alpha-1}\left(1+\frac{\alpha}{1-\alpha} y\right)^{-\alpha}\left[-\alpha\left(1+\frac{\alpha}{1-\alpha} y\right)+\alpha(1-y)\right]
$$

then $g^{\prime}(0)=0$ 
Let $y \in[0,1]$. Taylor formula leads to :

$$
\begin{aligned}
g(y) & =g(0)+g^{\prime}(0) y+\frac{g^{\prime \prime}(z)}{2} y^{2} \\
& =1-\frac{\alpha}{1-\alpha}(1-z)^{\alpha-2}\left(1+\frac{\alpha}{1-\alpha} z\right)^{-\alpha-1} \frac{y^{2}}{2}
\end{aligned}
$$

where $0<z<y$.

Since, $\alpha-2<0$ and $1-z$ belongs to $] 0,1\left[\right.$, then $(1-z)^{\alpha-2}>1$.

Similarly :

Therefore

$$
z<1 \Rightarrow 1+\frac{\alpha}{1-\alpha} z \leq 1+\frac{\alpha}{1-\alpha}=\frac{1}{1-\alpha}
$$

$$
\left(1+\frac{\alpha}{1-\alpha} z\right)^{-\alpha-1} \geq(1-\alpha)^{\alpha+1}
$$

and

$$
g(y) \leq 1-\frac{\alpha}{1-\alpha}(1-\alpha)^{\alpha+1} \frac{y^{2}}{2}=1-\alpha(1-\alpha)^{\alpha} \frac{y^{2}}{2} .
$$

\section{Proof of Theorem 2.8}

Suppose that (2.7) holds, i.e.

$$
1-\left(1-\theta_{0}\right)^{1 / n} \leq \frac{\alpha(1-\alpha)^{\alpha}}{2} .
$$

1) We claim that under (2.8) then $C P_{k, n, \alpha}^{*} \geq \theta_{0}$ where $\left.\theta_{0} \in\right] 0,1[$.

We set $\Pi^{k}\left(i_{0}, m\right)=1-\alpha+\alpha y$ where $\left.\left.y \in\right] 0,1\right]$. Obviously,

$$
y=\frac{\Pi^{k}\left(i_{0}, m\right)+\alpha-1}{\alpha}
$$

$y \leq 1$ and

$$
y \geq \sqrt{\frac{2\left[1-\left(1-\theta_{0}\right)^{1 / n}\right]}{\alpha(1-\alpha)^{\alpha}}} \Leftrightarrow 1-\Pi^{k}\left(i_{0}, m\right) \leq \alpha-\sqrt{\frac{2 \alpha\left[1-\left(1-\theta_{0}\right)^{1 / n}\right]}{(1-\alpha)^{\alpha}}}
$$

Consequently, (2.8) implies that

$$
y \geq \sqrt{\frac{2\left[1-\left(1-\theta_{0}\right)^{1 / n}\right]}{\alpha(1-\alpha)^{\alpha}}} .
$$

According to $(4.16),(4.17),(4.18)$ and Proposition 2.7 it follows that :

$$
\left(1-C P_{k, n, \alpha}^{*}\right)^{1 / n} \leq f\left(\Pi^{k}\left(i_{0}, m\right)\right)=g(y) \leq 1-\frac{\alpha(1-\alpha)^{\alpha}}{2} y^{2} .
$$

Using (4.20), we have successively :

$$
\begin{aligned}
& 1-\frac{\alpha(1-\alpha)^{\alpha}}{2} y^{2} \leq\left(1-\theta_{0}\right)^{1 / n} \\
& 1-C P_{k, n, \alpha}^{*} \leq 1-\theta_{0} \Rightarrow C P_{k, n, \alpha}^{*} \geq \theta_{0} .
\end{aligned}
$$

2 ) Suppose that $\alpha \approx 0$. Here we suppose that (2.9) holds, i.e.

$$
1-r \simeq\left\{\frac{1}{\left(m-i_{0}\right)(1+(m-1) q)^{k-1}}\left(\alpha-\sqrt{\frac{2 \alpha\left[1-\left(1-\theta_{0}\right)^{1 / n}\right]}{(1-\alpha)^{\alpha}}}\right)\right\}^{1 / k} .
$$


Since $\alpha-\sqrt{\frac{2 \alpha\left[1-\left(1-\theta_{0}\right)^{1 / n}\right]}{(1-\alpha)^{\alpha}}} \leq \alpha$, the former relation implies that $r$ is in the vicinity of 1 . Theorem 2.1 may be applied :

$$
\begin{aligned}
1-\Pi^{k}\left(i_{0}, m\right) & \simeq\left(m-i_{0}\right)(1+(m-1) q)^{k-1}(1-r)^{k} \\
& \simeq \alpha-\sqrt{\frac{2 \alpha\left[1-\left(1-\theta_{0}\right)^{1 / n}\right]}{(1-\alpha)^{\alpha}}} .
\end{aligned}
$$

This actually means that (2.8) holds. Step one of Theorem 2.8 gives $C P_{k, n, \alpha}^{*} \succeq \theta_{0}$.

\subsection{Proof of Proposition 2.9}

1) Recall that $1-e^{-x} \leq x$ for any positive $x$. Therefore

$$
1-\left(1-\theta_{0}\right)^{1 / n}=1-e^{\frac{1}{n} \ln \left(1-\theta_{0}\right)} \leq-\frac{\ln \left(1-\theta_{0}\right)}{n} .
$$

Since $n_{0}:=1+\left\lfloor-\frac{2 \ln \left(1-\theta_{0}\right)}{\alpha(1-\alpha)^{\alpha}}\right\rfloor$, we deduce immediately that if $n \geq n_{0}$ then condition (2.7) is satisfied. 2) Suppose that $n \geq 4 n_{0}$. We claim that

$$
\alpha-\sqrt{\frac{2 \alpha\left[1-\left(1-\theta_{0}\right)^{1 / n}\right]}{(1-\alpha)^{\alpha}}} \geq \frac{\alpha}{2} .
$$

Indeed (4.21), $n \geq 4 n_{0}$ and $n_{0} \geq-\frac{2 \ln \left(1-\theta_{0}\right)}{\alpha(1-\alpha)^{\alpha}}$ yield to :

$$
1-\left(1-\theta_{0}\right)^{1 / n} \leq-\frac{\ln \left(1-\theta_{0}\right)}{n} \leq-\frac{\ln \left(1-\theta_{0}\right)}{4 n_{0}} \leq \frac{\alpha(1-\alpha)^{\alpha}}{8} .
$$

We thus get :

$$
\sqrt{\frac{2 \alpha\left[1-\left(1-\theta_{0}\right)^{1 / n}\right]}{(1-\alpha)^{\alpha}}} \leq \frac{\alpha}{2}
$$

A straightforward calculation shows that (4.22) follows.

Recall that $r$ has been defined by (2.11), i.e.

$$
1-r=\left\{\frac{\alpha}{2\left(m-i_{0}\right)(1+(m-1) q)^{k-1}}\right\}^{1 / k} .
$$

Since $\alpha \approx 0$, then $1-r \rightarrow 0$. Consequently, Theorem 2.1 may be applied, using moreover the previous identity we obtain :

$$
1-\Pi^{k}\left(i_{0}, m\right) \sim\left(m-i_{0}\right)(1+(m-1) q)^{k-1}(1-r)^{k}=\frac{\alpha}{2} .
$$

As a result, we deduce from (4.22) that (2.8) holds. According to item 1 of Theorem 2.8 we can conclude that $C P_{k, n, \alpha}^{*} \geq \theta_{0}$. 


\section{References}

[1] K. C. Atwood and A. Norman. On the interpretation of multi-hit survival curves. Proc. Natl. Acad. Sci. USA, 35:696-709, 1949.

[2] T. Bastogne, R. Keinj, and P. Vallois. Multinomial model-based formulations of tcp and ntcp for radiotherapy treatment planning. Journal of Theoretical Biology, 279:55-62, 2011.

[3] T. Bastogne, R. Keinj, and P. Vallois. Tumor growth modeling based on cell and tumor lifespans. Submitted to Journal of Theoretical Biology, 2012.

[4] M. Blau and K. Altenburger. Uber einige Wirkungen von Strahlen. II. Z. Phys., 12(2):315-329, 1922.

[5] J. A. Crowther. Some considerations relative to the action of x-rays on tissue cells. In Proceedings of the Royal Society of London: Series B, pages 207-211, 1924.

[6] A. Dawson and T. Hillen. Derivation of the tumour control probability (tcp) from a cell cycle model. Computational and Mathematical Methods in Medicine, 7(2-3):121-141, 2006.

[7] Amir Dembo and Ofer Zeitouni. Large deviations techniques and applications. Jones and Bartlett Publishers, Boston, MA, 1993.

[8] F. Dessauer. Uber einige Wirkungen von Strahlen. I. Z. Phys., 12(1):38-47, 1922.

[9] H. A. Gay and A. Niemierko. A free program for calculating eud-based ntcp and tcp in external beam radiotherapy. Physica Medica, 23(115-125), 2007.

[10] D. E. Lea. Actions of Radiations on Living Cells. Cambridge University Press, Cambridge, UK, 1955.

[11] T. Nakagawa. Shock and Damage Models in Reliability Theory. Springer, Series in Reliability Engineering, 2007.

[12] A Niemierko and M. Goitein. Calculation of normal tissue complication probability and dosevolume histogram reduction schemes for tissues with a critical element architecture. Radiother Oncol., 20(3):166-76, March 1991.

[13] E. C. Pollard. Radiation inactivation of enzymes, nucleic acids and phage particles. Rev. Mod. Phys., 31(2):273-281, 1959.

[14] E. C. Pollard, W.R. Guild, F. Hutchinson, and R. B. Setlow. The direct action of ionizing radiation on enzymes and antigen. Progress in Biophysics, 5:72-108, 1955.

[15] N. Suntharaligam, E. B. Podgorsak, and J. H. Hendry. Radiation oncology physics : a handbook for teachers and students, chapter Basic Radiobiology, pages 485-504. E. B. Podgorsak, Vienna, Austria: International Atomic Energy Agency (IAEA), 2005.

[16] M Zaider and G N Minerbo. Tumour control probability: a formulation applicable to any temporal protocol of dose delivery. Physics in Medicine and Biology, 45(2):279-293, 2000. 